\title{
Constitutive model of AZ80+Ce alloy as-cast considering strain effect
}

\author{
Gang Chen, Jingjiang Nie, Kuanxin Liu \& Shunqi Zheng \\ Ningbo Branch of Ordnance Science Institute of China, Ningbo 315103, China
}

\begin{abstract}
In the present work, hot compression tests of AZ80+Ce alloy as-casted were carried out on a Gleeble-3500 thermo-mechanical simulator in temperatures range of $325-400{ }^{\circ} \mathrm{C}$ at intervals $25{ }^{\circ} \mathrm{C}$, at strain rates of $0.01,0.1,1$ and $10 \mathrm{~s}^{-1}$, respectively. The tests data at true strains of $0.1,0.2,0.3$ and 0.4 were employed for constitutive analysis following the Arrhenius equation. The material constants including $\mathrm{n}, \alpha, \beta$, and $\mathrm{Q}$ have been determined considering the strain compensation using the cubic polynomial fitting method. And then, incorporating the strain compensation on the constitutive equations for experimental alloy is established.
\end{abstract}

KEYWORD: magnesium alloy; Arrhenius-type constitutive model; strain-compensated

\section{INTRODUCTION}

Magnesium alloys have primary potential for lightweight structural application which are used in automotive and aerospace industries [1-3]. However, it exhibits poor workability at room temperature because of hexagonal close-packed crystal structure with limited number of slip systems [4-6]. In order to obtain high performance parts, simulation method is used for forming process optimization. The constitutive relationship describes the flow behavior of the materials, which is a very important input for material database construction.

The results of many studies carried out to establish the constitutive relationship show that there are some significant different in the constitutive characteristics of the different alloys [7-9]. Therefore, it is very important to understand the constitutive relationship [10-12]. Especially, the reliability of the simulation results and the accuracy of the decision based on the results are directly determined by precision of the constitutive relationship.

Constitutive relationship is usually described to be a mathematical expression containing some material constants in different forms $[7,10,12]$. The constants of the different magnesium alloys are modified by strain to improve the model precision in some studies [13-15]. However, very limited efforts have been paid to understand hot deformation behavior of AZ80+Ce Magnesium alloy considering the strain compensation.
In the present study, cubic polynomial fitting method is used to research the influence of strain, on the constants. The compensation effects of different constants are also investigated by correlation coefficient (R), respectively. A developed model describing the flow characteristics of the experimental alloy is also established

\section{EXPERIMENTAL}

Basing on the commercial AZ80 magnesium alloy elements, 1 percent Ce element was added to manufacture the experimental alloy bar as-cast by semicontinuous casting. The specimens for tests were machined with a height of $12 \mathrm{~mm}$ and a diameter of $10 \mathrm{~mm}$. The hot compression tests were carried out on a Gleeble-3500 thermo-mechanical simulator in temperature range of $325-400^{\circ} \mathrm{C}$, at constant strain rates of $0.01,0.1,1$ and $10 \mathrm{~s}^{-1}$. Each specimens were soaked at deformation temperature for $3 \mathrm{~min}$ to obtain homogenous microstructure before compression and were rapidly quenched in water after compression.

\section{CONSTITUTIVE MODEL AND CONSTANTS SOLUTION}

The stress-strain data obtained from compression tests under different deformation conditions were made some conversion using usual practice method. 
Those conditions can be used to determine the material constant in following equations when some one or two parameters are constants. Arrhenius type equation is one of the phenomenological constitutive models, representing the relationships among the flow stress, temperature and strain rate which is expressed as Eq. (1)-(4) [6-9].

$\dot{\varepsilon}=A f(\sigma) \exp (-Q / R T)$

$\dot{\varepsilon}=A_{1} \sigma^{n_{1}} \exp (-Q / R T) \quad$ for $\alpha \sigma<0.8$

$\dot{\varepsilon}=A_{2} \exp (\beta \sigma) \exp (-Q / R T) \quad$ for $\alpha \sigma>1.2$

$\dot{\varepsilon}=A_{3}[\sinh (\alpha \sigma)]^{n} \exp (-Q / R T) \quad$ for all $\sigma$

Where $\mathrm{R}$ is universal gas constant, $\mathrm{Q}$ is the activation energy. $A_{1}, A_{2}, A_{3}, n, n_{1}, \alpha$ and $\beta$ are experimentally determined constants, $\alpha=\beta / \mathrm{n}_{1}$, and $\sigma$ is the flow stress.

Zener-Holloman parameter $(\mathrm{Z})$ considering the effects of temperature and strain rate on material deformation behavior is expressed as Eqs. (5) - (7) [5, 7].

$Z=\dot{\varepsilon} \exp (Q / R T)$

$Z=A_{3}[\sinh (\alpha \sigma)]^{n}$

$$
A_{3}[\sinh (\alpha \sigma)]^{n}=\dot{\varepsilon} \exp (Q / R T)
$$

Eq. (2) and Eq. (3) are made equivalent deformation to obtain following linear relationships:

$$
\begin{aligned}
& \ln \dot{\varepsilon}=\ln \left(A_{1}\right)+n_{1} \ln \sigma-Q / R T \\
& \ln \dot{\varepsilon}=\ln \left(A_{2}\right)+\beta \sigma-Q / R T
\end{aligned}
$$

The slopes of the plots of Eq. (8) and Eq. (9) are the value of $\mathrm{n}_{1}$ and $\beta$, respectively. According to $\alpha=\beta / n_{1}, \alpha$ value can be calculated. The same method can be taken for Eq. (7), and the linear formula can be expressed as Eq.(10). Bring $(\alpha \sigma)$ into Eq. (10), the $1 / \mathrm{n}$ and $(\mathrm{Q} / \mathrm{RT})$ values can be calculated by linear fitting at different deformation conditions. According to the average intercept value $\left(\left(\ln \mathrm{A}_{3}-\right.\right.$ $\mathrm{Q} / \mathrm{TR}) / \mathrm{n})$, we can calculate $\ln \left(\mathrm{A}_{3}\right)$ value.

$\ln [\sinh (\alpha \sigma)]=\frac{1}{\mathrm{n}} \ln \dot{\varepsilon}+\frac{Q}{n R T}-\frac{\ln \left(A_{3}\right)}{\mathrm{n}}$

Tab 1 values of materials constants at various strains

\begin{tabular}{l|l|l|l|l|l}
\hline \multirow{2}{*}{ True strain } & \multicolumn{4}{l}{ Material constants } \\
\cline { 2 - 6 } & $\beta$ & $\alpha$ & $\mathrm{n}$ & \multicolumn{1}{l}{$\mathrm{Q} \mathrm{A}_{3}$} \\
\hline 0.1 & 0.08932 & 0.010914 & 6.21995 & 309.76 & 70.63 \\
0.2 & 0.07677 & 0.011241 & 5.14064 & 308.08 & 70.29 \\
0.3 & 0.08498 & 0.012193 & 5.23300 & 307.77 & 70.39 \\
0.4 & 0.09885 & 0.013353 & 5.55819 & 308.23 & 70.73 \\
0.5 & 0.01144 & 0.014440 & 5.95621 & 308.79 & 71.10 \\
\hline
\end{tabular}

The same procedure was also used to calculate the values of materials constants in the strains range of $0.1-0.5$, at step of 0.1 , respectively.
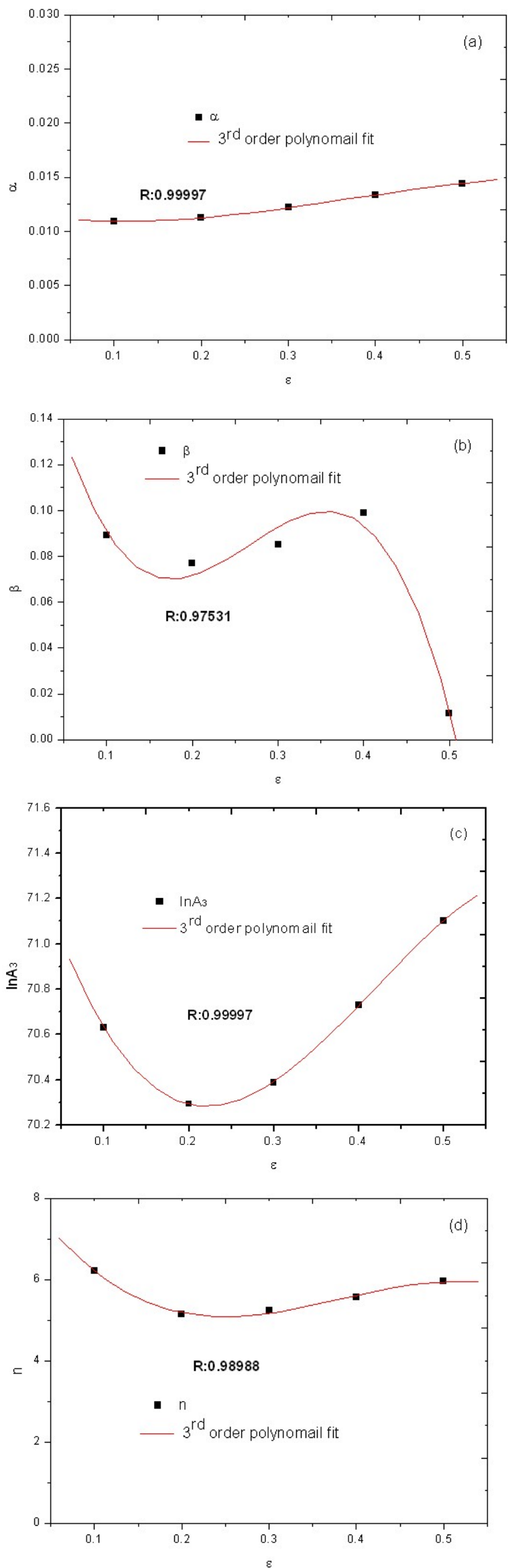


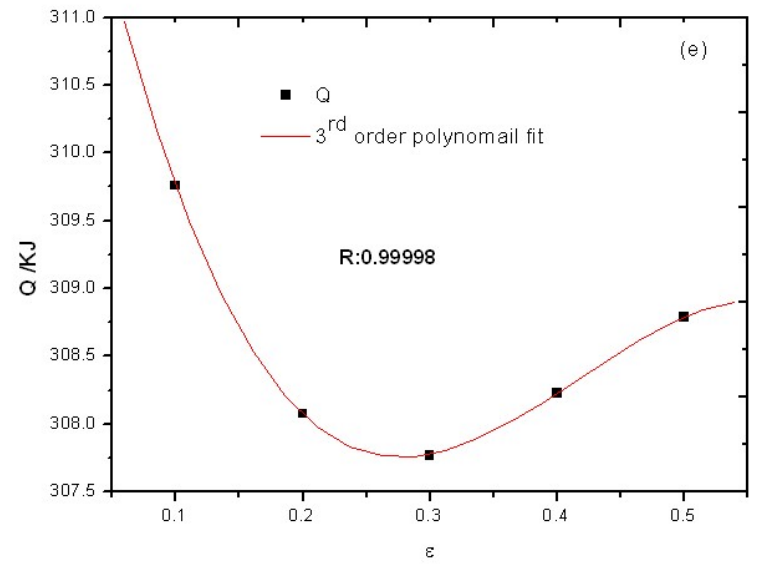

Fig. 1 Variation of (a) $\alpha$, (b) $\beta$, (c) $\ln \mathrm{A} 3$, (d) $\mathrm{n}$ and (e) $\mathrm{Q}$ with true strain $(0.1-0.5)$

It can be seen clearly that strain has great impact on constant values which are given in Tab.1. Hence, the Arrhenius type equation of the experimental alloy has not a satisfactory accuracy, which is established just using the average values at different strains. Some studies results show that the material constants which are polynomial functions of strain can improve the model accuracy [16-20]. Hence, cubic polynomial fitting method is used to find out the

$$
\begin{aligned}
& \dot{\varepsilon}=A_{3}(\varepsilon)[\sinh (\alpha(\varepsilon) \sigma)]^{n(\varepsilon)} \exp (-Q(\varepsilon) / R T) \\
& \alpha(\varepsilon)=0.01152-0.01197 \varepsilon+0.06469 \varepsilon^{2}-0.05817 \varepsilon^{3} \\
& \beta(\varepsilon)=0.2112-1.91639 \varepsilon+8.1239 \varepsilon^{2}-10.16981 \varepsilon^{3} \\
& \ln A_{3}(\varepsilon)=71.606-13.61224 \varepsilon+41.89929 \varepsilon^{2}-33.38333 \varepsilon^{3} \\
& \mathrm{n}(\varepsilon)=8.7867-35.38118 \varepsilon+105.181 \varepsilon^{2}-91.57025 \varepsilon^{3} \\
& \mathrm{Q}(\varepsilon)=313.4808-49.50119 \varepsilon+133.69643 \varepsilon^{2}-106.91667 \varepsilon^{3}
\end{aligned}
$$

relationship between different constants and strain, respectively. The fitting results are shown in Fig. 1. The change characteristic of different constants also can be seen. The $\alpha$ value increases with increasing strain rapidly. The $n, \ln \mathrm{A}_{3}$ and $\mathrm{Q}$ values decrease firstly and then increase with increasing strain. The $\beta$ values show " $N$ " shape changing tendencies with increasing strain.

According to the fitting curves, the fitting method equations were given in Eq. (11). The fitting results show that $3^{\text {rd }}$ order polynomials can describe the influence of material constants with good correlation and generalization basing on the correlation coefficient which are 0.99997, 0.97531, 0.99997, 0.98988 and 0.99998, respectively in Fig. 1 (a)-(e).

$$
\begin{aligned}
& \alpha(\varepsilon)=0.01152-0.01197 \varepsilon+0.06469 \varepsilon^{2}-0.05817 \varepsilon^{3} \\
& \beta(\varepsilon)=0.2112-1.91639 \varepsilon+8.1239 \varepsilon^{2}-10.16981 \varepsilon^{3} \\
& \ln \mathrm{A}_{3}(\varepsilon)=71.606-13.61224 \varepsilon+41.89929 \varepsilon^{2}-33.38333 \varepsilon^{3} \\
& \mathrm{n}(\varepsilon)=8.7867-35.38118 \varepsilon+105.181 \varepsilon^{2}-91.57025 \varepsilon^{3} \\
& \mathrm{Q}(\varepsilon)=313.4808-49.50119 \varepsilon+133.69643 \varepsilon^{2}-106.91667 \varepsilon^{3}
\end{aligned}
$$

Using the fitting equations, the straincompensated Arrhenius-type constitutive model can express as following:

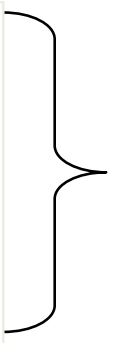

\section{SUMMARY}

Hot compression tests were conducted in temperatures range of $325-400^{\circ} \mathrm{C}$, at strain rates of $0.01,0.1$, 1 and $10 \mathrm{~s}^{-1}$. Strain has shown significant influence on materials constants at different true strain (0.10.5 , at step of 0.1 ). Fitting curves show that the $\alpha$ value increases with increasing strain rapidly and the $\mathrm{n}, \ln \mathrm{A}_{3}$ and $\mathrm{Q}$ values decrease firstly and then increase with increasing strain. The $\beta$ values show " $N$ " shape changing tendencies with increasing strain. The constitutive mode was compensated by different constants fitting equations. The results show that cubic polynomials can $\mathrm{f}$ present the influence of strain on material constants with good correlation and generalization in terms of the adjusted coefficients of determination.

\section{REFERENCES}

[1] Hui zhong LI, Xiaoyan WEI, Jie OUYANG et al. Trans. Nonferrous Met. Soc. China, 2013, 23: 3180.
[2] Xiang-heng XIA, Ming CHEN, Yongjin LU et al. Trans. Nonferrous Met. Soc. China, 2013, 23:3186.

[3] LI zhao-zhi, YANG yaqin, ZHANG Zhimin. Trans. Nonferrous Met. Soc. China, 2008, 18: 156.

[4] ZHANG J, FANG C, YUAN F, LIU C. Mater. Des, 2011, 32 (4): 1783.

[5] M. Sarebanzadeh, R.Mahmudi, R.Roumina. Mater. Sci.Eng. A, 2015, 637:155.

[6] Hamed Mirzadeh. Mech. Mater., 2014, 77: 80.

[7] H. Mirzadeh, A. Najafizadeh. Mater. Sci. Eng. A, 2010, 527:1160.

[8] H. Mirzadeh, M.H. Parsa, D. Ohadi. Mater. Sci. Eng. A, 2013, 569: 54.

[9] Chen Wei, Gang Chen, Jing Zhai et al. Adv. Mater. Res., 2014, 968:3.

[10] Y.C. Lin, Yu-Chi Xia, Xiao-Min Chen, et al. Comput. Mater. Sci. 2010, 50:227.

[11] V. Senthilkumar, A. Balaji, R. Narayanasamy. Mater. Des., 2012, 37: 102.

[12] Songyi Chen, Kanghua Chen, Guosheng Peng et al. J Alloys Compd., 2012, 537: 338.

[13] M. Rajamuthamilselvan, S. Ramanathan. J Alloys Compd., 2011, 509: 948

[14] A. Abbasi-Bani, A. Zarei-Hanzaki, M.H. Pishbin et al. Mechan. Mater, 2014, 71:52.

[15] Hamed Mirzadeh. Mater. Chem. Phys., 2015, 152:123. 
[16] Nengping Jin, Hui Zhang, Yi Han et al. Mater Charact., 2009, 60: 530

[17] Dipti Samantaray, Sumantra Mandal, A.K. Bhaduri, Comput. Mater. Sci., 2009, 47:568.

[18] C. Phaniraj, Dipti Samantaray, Sumantra Mandal et al. Mater. Sci.Eng. A, 2011, 528: 6066

[19] Dipti Samantaray, Sumantra Mandal, M. Jayalakshmi et al. Mater. Sci. Eng. A, 2014, 598: 368

[20] Dipti Samantaray, Sumantra Mandala, Vinod Kumar et al. Mater. Sci .Eng. A, 2012, 512: 236 